\title{
Regulatory framework in 3D printing
}

\author{
Aishwarya Bhargav ${ }^{* 1}$ \\ ${ }^{1}$ Department of Mechanical Engineering, National University of Singapore, Singapore \\ *Author for correspondence: Aishwarya.bhargav@u.nus.edu
}

\begin{abstract}
3D printing or additive manufacturing has induced a paradigm change in the healthcare space. Medical devices, drugs and even cells may be processed using 3D printing. Despite the patient-specific advantages that 3D printing offers, several companies hesitate to incorporate it into the manufacturing process owing to the ambiguity in the regulatory framework. There exists confusion on whether the printer itself needs to be treated as a medical device and regulated or whether it is the end product that needs to be regulated. This article aims to elaborate the regulatory pathways that may be considered for 3D printed devices.
\end{abstract}

First draft submitted: 29 July 2017; Accepted for publication: 11 August 2017; Published online: 24 October 2017

Keywords: 3D printing • medical devices • regulation

\section{Drugs \& biologics}

In 2015, the US FDA granted approval for 'Spritam', a 3D printed drug that could potentially be used to treat epilepsy [1]. It was found that 3D printing enabled the creation of thin layers of drug thereby assisting in the faster dissolution of the pill. In this case, while 3D printing aids in improving the efficacy of the drug; it is the chemical reaction owing to the composition of the drug that finally causes the required effect. Hence in this scenario, both the chemical composition as well as the process of manufacturing play a vital role in maintaining the efficiency of the drug. Hence, instead of regulating the chemical composition of the drug or regulating the process of fabrication, the final product, that is, $3 \mathrm{D}$ printed spritam is brought under the regulatory framework as a drug.

Similarly, cells and other active components are regulated as biologics owing to the mechanism of action, which is biological in nature.

\section{Common patient-specific devices}

Medium \& high risk

Orthopedic implants, heart valves and dental implants are among the devices that are commonly fabricated using $3 \mathrm{D}$ printing owing to the ability of $3 \mathrm{D}$ printing to create customized implants based on the anthropometric characteristics of the patient [2]. In such a scenario, the 3D printed implant is sold by a healthcare service provider to the patient. The efficiency of the implant depends on several factors such as the dimensional accuracy and surface finish of the implant. Here, the efficiency of the implant depends on the skill of the operator (producing the implant), the $3 \mathrm{D}$ printer itself and the interface or software controlling the $3 \mathrm{D}$ printer. In this scenario, as the manufacturer of the 3D printer does not interact with the patient directly, it becomes highly imperative that the 3D printer is accurate in order to reduce the chance of error. Hence, the 3D printer needs to be regulated and checked for efficiency. However, as it is the final implant that will be used, there is a need to regulate the final application.

For example, a ' $3 \mathrm{D}$ printed hip joint' is a class III medical device that possesses high risk. Failure may occur either owing to poor design of the implant, poor manufacturing or material failure. Hence the entire device, including the material and manufacturing process needs to be regulated.

\section{Low-risk medical devices}

Assistive aids such as 3D printed grabber, customized tooth brushes, etc. present low risk to the patient. Such devices require good manufacturing processes to be followed while manufacturing. These devices are exempted from regulation owing to the low level of risk that the patient is subjected to.

Future : Medicine 


\section{Humanitarian \& custom device exemption}

In 2013, a 6-week-old baby was fitted with a 3D printed artificial windpipe to assist in breathing [3]. This device did not require regulation owing to humanitarian exemption which allows a device to bypass the lengthy regulatory process if a patient needs it immediately for survival. Similarly, devices used to treat patients who suffer from exceptionally rare conditions are often granted exemption owing to the rarity of the condition that makes clinical trials impossible owing to the absence of a sufficient sample size to reach a conclusion on whether the device is suitable for the market.

\section{Concerns regarding 3D printing}

In my opinion, some of the concerns regarding 3D printing for medical applications are discussed as follows:

- The versatility of 3D printing enables the processing of a wide range of materials. This allows for the misuse of 3D printers. Drugs can be assembled at the molecular level. While this is very useful to enhance the quality of medication, there is a possibility that drugs such as cocaine may be assembled from scratch using a 3D printer.

- 3D printing has given rise to a do-it-yourself culture, which can cause considerable damage in the case of self-treatment. Articles regarding how to print your own implant or braces are available online. This may prove to be disastrous if the patient is unaware of proper protocols to be followed.

\section{Conclusion}

The benefits of $3 \mathrm{D}$ printing far outweigh the negatives that $3 \mathrm{D}$ printing possesses. $3 \mathrm{D}$ printing as a manufacturing technique for medical devices; drugs and biologics is here to stay in the long run. Hence, it is of importance to optimize and improve the 3D printing process in order to achieve the best possible outcomes for the patient.

$3 \mathrm{D}$ printing is after all a manufacturing technique like casting or welding. The outcomes of the process depends more on the material and the skill of the person operating the process. Just like other manufacturing techniques may be exploited for negative uses, 3D printing may also be exploited for the wrong purposes. Hence, the negatives of $3 \mathrm{D}$ printing may not be attributed to the process.

The regulatory framework that needs to be applied in 3D printing is similar to that of conventionally manufactured devices, except for the fact that 3D printed devices are used almost instantaneously after fabrication, thereby not allowing for prolonged quality assurance processes. Hence, the 3D printing process should be optimized such that quality control is incorporated into the manufacturing process itself. Thereby reducing the need for quality assurance and testing post-manufacturing.

\section{Financial \& competing interests disclosure}

The author received support from the National University of Singapore Research Scholarship.

No writing assistance was utilized in the production of this manuscript.

\section{References}

1 The FDA just approved the first 3D printed drug (January 10, 2017). (2017). http://medicalfuturist.com/the-fda-just-approved-the-first-3d-printed-drug/

2 Bhargav A, Sanjairaj V, Rosa V, Feng LW, Fuh YH J. Applications of additive manufacturing in dentistry: a review. J. Biomed. Mater. Res. B Appl. Biomater. 10.1002/jbm.b.33961 (2017) (Epub ahead of print).

33 D printed windpipe gives infant breath of life. (2017).

www.nature.com/news/3-d-printed-windpipe-gives-infant-breath-of-life-1.13085 